\title{
Desempenho da Ultra-sonografia Pré-natal no Diagnóstico de Cromossomopatias Fetais em Serviço Terciário
}

\author{
Performance of Prenatal Ultrasound in the Diagnosis of Fetal Chromosomal \\ Abnormalities in a Tertiary Center
}

\begin{abstract}
Ricardo Barini ${ }^{1}$, Juliana Horschutz Stella ${ }^{2}$, Sara Toseti Ribeiro ${ }^{2}$, Fátima BotcherLuiz ${ }^{3}$, Eduardo Valente Isfer ${ }^{4}$ Rita de Cassia Sanchez ${ }^{4}$, Aníbal Faúndes ${ }^{5}$, João Luiz Pinto e Silva ${ }^{5}$
\end{abstract}

\section{RESUMO}

Objetivo: determinar o desempenho da ultra-sonografia para detecção de gestações que apresentem risco de anomalia cromossômica fetal.

Método: estudamos 436 pacientes submetidas a cariótipo fetal entre março de 1993 e março de 1998. Destas, 277 gestantes foram submetidas à coleta de material fetal para estudo citogenético por ultra-sonografia alterada e 159 por ansiedade parental, apresentando ultrasonografia morfológica normal. Foram avaliadas a sensibilidade e a especificidade da ultrasonografia utilizando o cariótipo fetal como padrão-ouro. Foi calculado o risco relativo de anomalia cromossômica apresentado segundo o sistema acometido à ultra-sonografia e os riscos de acordo com a presença de uma ou mais malformações, utilizando-se o pacote de análise estatística Epi-Info 6.0.

Resultados: observamos que o risco relativo para cromossomopatia fetal foi de 89 para as malformações de face, 53 para malformações de parede abdominal e aparelho cardiovascular, 49,6 para malformações de pescoço, 44,6 para malformações de membros, 42,4 para malformações de pulmão, 32,7 para malformações de trato gastrointestinal, 27,4 para malformações de sistema nervoso central e 23,0 para malformações de trato urinário. Não foi possível o cálculo de risco para malformações de genitais, tórax, coluna e músculo-esqueléticas, por terem ocorrido em um pequeno número de casos. A presença de uma alteração ultrasonográfica isolada se associa a risco relativo 7,82 vezes maior de cromossomopatia, ao passo que a associação de alterações morfológicas tem risco 33,8 vezes maior de cromossomopatia. Conclusões: conclui-se que a ultra-sonografia apresentou bom desempenho na detecção de gestações de risco para cromossomopatia.

PALAVRAS-CHAVE: Ultra-sonografia. Cromossomopatias fetais. Diagnóstico pré-natal. Cariótipo. Malformação fetal.

\section{Introdução}

Com o avanço nos cuidados na área da saúde pública e a melhoria das condições socioeconômicas e dos cuidados médicos, a mortalidade in-

Centro de Atenção Integral à Saúde da Mulher (CAISM) da Universidade Estadual de Campinas (UNICAMP) e Centro de Diagnóstico Pré-Natal e Medicina Fetal (FETUS) em São Paulo.

Correspondência:

Ricardo Barini

Rua Francisco Humberto Zuppi, 500

13083-350 - Campinas - SP

Fax/fone: (19) 289-4161 / (19) 788-9304

e-mail: barini@caism.unicamp.br fantil vem diminuindo ao longo dos anos na maior parte dos países, inclusive no Brasil ${ }^{1,2}$. Porém, após uma queda significativa inicial do indice houve uma progressiva estagnação, mesmo em países desenvolvidos $^{3}$, e na investigação das possiveis causas deste fenômeno, as malformações fetais aparecem como a principal causa de óbito na infância dos países do primeiro mundo. Cerca de 2 a $3 \%$ dos recém-nascidos são portadores de uma ou mais malformações congênitas, sendo responsáveis por $20 \%$ da mortalidade neonatal e 30 a $50 \%$ da mortalidade perinatal nos países desenvolvidos ${ }^{4}$.

As malformações congênitas constituem uma das dez primeiras causas de mortalidade in- 
fantil. A posição relativa das anomalias congênitas como causa de óbito é tão mais elevada quanto menor a mortalidade infantil ${ }^{5}$. Estudos de morbidade em crianças indicam que as enfermidades genéticas e os defeitos congênitos representam 10-25\% das internações em estabelecimentos de assistência terciária em alguns centros urbanos da América Latina.

$\mathrm{Na}$ América Latina, as informações sobre a freqüência de defeitos congênitos são ainda fragmentadas, por deficiência no diagnóstico e de estatísticas de saúde confiáveis. Dados oriundos de estudos locais e do Estudo Colaborativo Latino-Americano de Malformações Congênitas (ECLAMC) confirmam que os defeitos congênitos da região têm freqüência similar à de outros lugares ${ }^{3,5}$.

Nos últimos anos tem surgido em todo o mundo o interesse de identificar anomalias cromossômicas fetais principalmente por meio de métodos não invasivos. Têm sido utilizados com mais freqüência os rastreamentos por grupo de risco, bioquímico e ultra-sonografia ${ }^{5,6}$.

Os progressos na tecnologia da ultrasonografia têm contribuído para aumento da detecção de fetos com anomalias estruturais em populações de baixo risco ${ }^{7,8}$, tornando-se paulatinamente parte da rotina dos cuidados pré-natais.

O exame de ultra-sonografia permite identificar diretamente alterações morfológicas individuais, sinais indiretos relacionados (crescimento fetal restrito e alterações do volume de líquido amniótico, entre outras), além das malformações associadas, que apresentam estreita correlação com quadros sindrômicos. Com a aquisição de maior experiência na identificação da morfologia fetal e o acesso simplificado a estudos citogenéticos, cresceu o conhecimento sobre a correspondência entre achados morfológicos alterados à ultra-sonografia e alterações específicas do cariótipo $^{9-12}$.

Apesar de controvérsias remanescentes, recomenda-se que a primeira ultra-sonografia para triagem de anomalias morfológicas deve ser realizada entre a $16^{\mathrm{a}}$ e a $22^{\mathrm{a}}$ semana de gestação e as posteriores entre a 26a e a 28 a $^{\text {a }}$ semana e a $32^{\text {a }}$ e a $34^{\mathrm{a}}$ semana. Há uma crescente tendência atual de incluir-se um exame entre a 10 a e a 14a semana de gestação para medida de translucência nucal (quantidade de líquido situada entre a pele e o tecido mole que circunda a coluna cervical). Quando esta agenda não for possivel de se cumprir, aconselha-se a realização de pelo menos um exame ultra-sonográfico entre a $26^{\text {a }}$ e a 28 a semana, considerado o melhor período para avaliação estrutural fetal, incluindo o diagnóstico de displasias esqueléticas e malformações do trato gastrointestinal ${ }^{4,13}$.
O objetivo deste trabalho é determinar o desempenho do exame ultra-sonográfico em identificar fetos com risco de apresentar anomalias cromossômicas, estabelecendo o risco relativo que as alterações morfológicas específicas apresentam para identificar alterações cromossômicas fetais e se o número de malformações identificadas em cada feto modifica o risco relativo para cromossomopatias.

\section{Pacientes e Métodos}

Trata-se de um estudo para validação da ultra-sonografia como teste diagnóstico. O tamanho amostral foi calculado por meio de fórmula para estudos de teste diagnóstico, utilizando como base os dados do estudo de Nicolaides et al. ${ }^{14}$, que tinha como objetivo descrever marcadores ultrasonográficos de anomalias cromossômicas.

Participaram do estudo 436 pacientes atendidas no Centro de Atenção Integral à Saúde da Mulher (CAISM) e no Centro de Diagnóstico PréNatal e Medicina Fetal (FETUS-SP) entre janeiro de 1994 e janeiro de 1998. Todas foram submetidas à coleta de material para realização de cariótipo fetal, com idade gestacional variando entre 14 e 38 semanas.

As gestantes foram selecionadas a partir dos registros do Setor de Medicina Fetal dos serviços citados. Foram incluídas aquelas atendidas no periodo e que se submeteram ao exame citogenético por diagnóstico ultra-sonográfico de alteração morfológica fetal e que constituíram o grupo de estudo, assim como aquelas com gestações morfologicamente normais à ultra-sonografia, submetidas à coleta de material por motivos outros que não envolviam doenças fetais, que constituíram o grupo controle. Os critérios de exclusão foram os prontuários com dados incompletos, suspeita de infecção congênita e hidropisia fetal imune. As pacientes foram submetidas à amniocentese ou cordocentese para diagnóstico genético.

O projeto foi aprovado pela Comissão de Ética em Pesquisa da instituição. Os exames de ultrasonografia foram realizados por médicos da equipe das duas instituições, utilizando equipamento marca Toshiba, modelo SAL140. Os exames de cariótipo foram realizados por dois laboratórios, o Laboratório de Procedimentos Especializados do CAISM e pelo Centro Médico Especializado S/C Ltda., ambos em Campinas, São Paulo.

Os dados foram analisados por meio do pacote de análise estatística Epi-Info 6.0. Foram avaliados sensibilidade, especificidade e valores preditivos positivo (VPP) e negativo (VPN) de alterações 
morfológicas fetais à ultra-sonografia, além do cálculo da prevalência de cromossomopatia na população estudada. Foi realizada a estimativa do risco relativo por categoria de acometimento morfológico fetal. Foram utilizados os testes de $\chi^{2}$ para tabelas $2 \times 2$ e o teste exato de Fisher quando necessário.

\section{Resultados}

Nos 436 casos submetidos a exame ultrasonográfico e cariótipo, 277 apresentavam morfologia alterada e 159 eram morfologicamente normais. Ocorreram alterações cromossômicas em 9,1\% das gestações estudadas, sendo que $97,5 \%$ destas alterações concentraram-se no grupo com ultra-sonografia alterada. A freqüência de alterações cromossômicas nas pacientes com ultra-sonografia normal foi de $0,6 \%$, e com as alteradas foi de $14 \%$.

O único caso com ultra-sonografia normal e aberração cromossômica presente foi de um feto com trissomia do cromossomo 21.

A sensibilidade da ultra-sonografia alterada para identificar os indivíduos com aberração cromossômica na amostra foi de 97,5\%. Apesar da alta capacidade de detecção de gestações com aberração cromossômica, a ultra-sonografia apresentou alta taxa de falso-positivos, com baixo VPP (Tabela 1).

Tabela 1 - Desempenho da ultra-sonografia no diagnóstico de cromossomopatias.

\begin{tabular}{lccc}
\hline Ultra-sonografia & \multicolumn{3}{c}{ Cariótipo } \\
\hline & Alterado & Normal & Total \\
Alterada & 39 & 238 & 277 \\
Normal & 1 & 158 & 159 \\
Total & 40 & 396 & 436 \\
\hline Sensibilidade $=97,5 \%$ & Especificidade $=39,8 \%$ & & \\
VPP $=14 \%$ & VPN $=99,3 \%$ & Prevalência $=9,1 \%$
\end{tabular}

Com exceção de malformações em que o número de casos foi pequeno, de seis ou menos anomalias, como as de genitais, hidropisia não imune, coluna e músculo-esqueléticas, todas as demais apresentaram risco de cromossomopatia.

Os riscos relativos mais elevados ocorreram para anomalias morfológicas de face, parede abdominal, cardiovasculares, pescoço, membros e pulmão. Alterações gastrointestinais, do sistema nervoso central (SNC) e urinárias tiveram também riscos relativos elevados, entre 32 e 23. Os intervalos de confiança foram amplos, porém mantiveram valores elevados mesmo quando considerado o limite inferior (Tabela 2 ).
Tabela 2 - Risco relativo de cromossomopatia segundo o sistema comprometido no exame ultra-sonográfico.

\begin{tabular}{lccc}
\hline Anomalia & Prevalência & Risco relativo & IC 95\% \\
\hline Ausente & $1 / 163$ & 1 & - \\
Face & $14 / 25$ & 89,0 & $12,2-647,7$ \\
Parede abdominal & $8 / 24$ & 53,0 & $6,9-405,2$ \\
Cardiovascular & $9 / 27$ & 53,0 & $6,9-401,6$ \\
Pescoço & $5 / 16$ & 49,6 & $6,1-399,5$ \\
Membros & $12 / 43$ & 44,3 & $5,9-331,8$ \\
Pulmão & $4 / 15$ & 42,4 & $5,0-355,5$ \\
Trato gastrointestinal & $7 / 34$ & 32,7 & $14,6-257,4$ \\
Sistema nervoso central & $25 / 145$ & 27,4 & $3,7-199,7$ \\
Trato urinário & $9 / 62$ & 23,0 & $2,9-178,4$ \\
RCIU & $3 / 12$ & 2,8 & $1,0-8,0$ \\
Líquido amniótico & $11 / 61$ & 2,3 & $1,2-4,4$ \\
Hidropisia & $4 / 28$ & 1,6 & $0,6-4,2$ \\
Genitália & $0 / 3$ & - & - \\
Coluna & $0 / 4$ & - & - \\
Músculo-esqueléticas & $0 / 8$ & - & - \\
\hline RCIU, & & &
\end{tabular}

$\mathrm{RCIU}$, retardo de crescimento intra-uterino.

Dentre todos os diagnósticos ultrasonográficos, ocorreram cinco casos em que uma anomalia isolada esteve relacionada ao diagnóstico de cromossomopatia. As malformações fetais diagnosticadas nestes casos foram: dois fetos com diagnóstico ultra-sonográfico de hidrocefalia com resultado citogenético de trissomia do 13; um feto com diagnóstico ultra-sonográfico de holoprosencefalia com diagnóstico citogenético de triploidia; um feto com diagnóstico ultra-sonográfico de hidropisia com diagnóstico citogenético de síndrome de Turner; um feto com diagnóstico ultra-sonográfico de higroma cístico de pescoço com diagnóstico citogenético de trissomia do 21 .

O risco relativo de cromossomopatia foi cerca de quatro vezes maior quando houve diagnóstico de mais de uma anomalia morfológica fetal à ultra-sonografia (Tabela 3).

Tabela 3 - Risco relativo de cromossomopatia na multiplicidade de anomalias encontradas à ultra-sonografia.

\begin{tabular}{lccc}
\hline Anomalia & Prevalência & Risco relativo & IC 95\% \\
\hline Ausente & $1 / 159$ & 1 & \\
Única & $6 / 122$ & 7,8 & $0,9-64,1$ \\
Múltipla & $33 / 155$ & 33,8 & $4,6-244,4$ \\
\hline
\end{tabular}

As principais malformações identificadas pelo ultra-som e as aneuploidias diagnosticadas nesta casuística estão resumidas (Tabela 4). 
Tabela 4 - Aneuploidias e principais sinais ultra-sonográficos identificados no grupo de estudo.

\begin{tabular}{|c|c|c|c|c|}
\hline \multirow{2}{*}{$\begin{array}{l}\text { Anomalias } \\
\text { cromossômicas mais } \\
\text { freqüentes ( } n=\text { total) }\end{array}$} & \multicolumn{4}{|c|}{$\begin{array}{l}\text { Principais sinais ultra-sonográficos identificados } \\
\text { (freqüência de aparecimento, isolado ou não). }\end{array}$} \\
\hline & Sistema nervoso central & Face & Tórax/abdome & Outras \\
\hline Trissomia 21 & Hidrocefalia (2), & Hipotelorismo (1) & Malformação cardíaca (1) & Hidropisia (3) \\
\hline $\mathrm{n}=8$ & Cisto de fossa posterior (1) & & & Higroma cístico (1) \\
\hline Trissomia 18 & Hidrocefalia (4) & Hipotelorismo (4) & Onfalocele (4) & Polidrâmnio (3) \\
\hline \multirow[t]{3}{*}{$n=16$} & Holoprosencefalia (2) & Exoftalmo (1) & Hérnia diafragmática (1) & Alças ecogênicas (1) \\
\hline & Cisto de fossa posterior (3) & Fenda palatina (3) & Malformação cardíaca (3) & Malformação de pés (8) \\
\hline & Hipoplasia de vérmix cerebelo (1) & & Hidronefrose (1) & Crescimento intra-uterino restrito (3) \\
\hline Trissomia 13 & Hidrocefalia (3) & Hipotelorismo (2) & Malformação cardíaca (3) & Malformação de pés (1) \\
\hline \multirow[t]{2}{*}{$n=7$} & Holoprosencefalia (3) & Microftalmia (1) & Displasia renal (1) & Artéria umbilical única (1) \\
\hline & Microcefalia (2) & Fenda palatina (3) & Onfalocele (1) & \\
\hline Monossomia X & Hidrocefalia (1) & & & Hidropsia (2) \\
\hline \multirow[t]{2}{*}{$\mathrm{n}=2$} & & & & Higroma cístico (1) \\
\hline & & & & Oligoâmnio (1) \\
\hline Triploidia & Microcefalia (1) & & & Oligoâmnio (1) \\
\hline \multirow[t]{2}{*}{$n=2$} & Holoprosencefalia (1) & & & Malformação de pés (1) \\
\hline & & & & Crescimento intra-uterino restrito (1) \\
\hline
\end{tabular}

Observação: não estão incluídos todos os casos de aneuploidia identificados. Constam desta tabela os sinais ultra-sonográficos e as aneuploidias mais freqüentes identificados neste estudo.

\section{Discussão}

Este estudo tem por objetivo avaliar o desempenho da ultra-sonografia como método diagnóstico na maioria das vezes em pacientes previamente selecionadas por encaminhamento de outros serviços à instituição universitária, na detecção de alterações cromossômicas fetais. Não se trata portanto de um estudo que avalie o desempenho da ultra-sonografia como método de rastreamento de cromossomopatias. Se esse fosse o objetivo do trabalho, seu desenho partiria não de casos conhecidos como portadores de alterações ultrasonográficas, mas sim de uma amostra representativa da população, com uma prevalência de alterações cromossômicas mais próxima da esperada. Neste trabalho a alta prevalência de alterações cromossômicas torna inadequada a proposição de se extrapolar os resultados aqui determinados e transformar a ultra-sonografia como método de rastreamento populacional. Ressaltamos que o exame de ultra-sonografia prévio a qualquer procedimento invasivo permite uma melhor certeza diagnóstica quanto à possibilidade de serem identificadas anomalias fetais que se relacionem com alterações cromossômicas.

Apesar das considerações iniciais, observamos que a ultra-sonografia se revelou um bom método para o rastreamento de anomalias cromossômicas na amostra populacional avaliada. Os re- sultados mostram que a ultra-sonografia foi um exame altamente sensível, capaz de detectar anomalias estruturais que se correlacionam com risco de cromossomopatias. A especificidade foi baixa, com alta taxa de falso-positivos. Os valores de sensibilidade e especificidade encontrados foram semelhantes aos descritos em estudos mais recen$\operatorname{tes}^{10,14-17}$.

Observamos ainda um alto VPN, ou seja, uma vez que o exame ultra-sonográfico não detecte anomalia fetal, a possibilidade de ocorrência de cromossomopatia é baixa. Ao contrário da amostra utilizada, em populações não préselecionadas sem fatores de risco para cromossomopatias, o VPN provavelmente seria maior. Como os valores preditivos de um teste dependem da prevalência da doença na população em questão, o VPP é variável na bibliografia consultada, com relatos de valores desde 0,5 até $30 \%$ conforme características da amostra estudada ${ }^{2,12,15}$.

A freqüência de anomalias citogenéticas em fetos malformados encontrados neste estudo, de $9,1 \%$, é próxima à relatada na literatura, que refere valores entre 14 e $20 \%$ de cromossomopatias em fetos com alterações morfológicas à ultrasonografia ${ }^{2,14}$.

Sabemos, entretanto, que as alterações encontradas apresentam diferentes riscos de cromossomopatias, o que justifica a necessidade de se determinar o risco específico para cada tipo de malformação fetal, assim como para um determi- 
nado grupo de alterações, ou ainda para determinadas associações específicas.

Da mesma maneira que o observado por Carrera et al. ${ }^{7}$ e Plaza et al. ${ }^{15}$, as anomalias estruturais fetais mais freqüentes foram as de SNC. Cisneros et al. $^{2}$, relatam estas alterações como a quarta em freqüência em sua população, porém eram casos de malformações isoladas. Tanto Carrera et al. ${ }^{7}$ quanto Plaza et al. ${ }^{15}$ relatam uma freqüência de cerca de $20 \%$ destas malformações, ao passo que na presente amostra este valor foi $50 \%$ maior (33\% de todas as anomalias).

Avaliando o risco de cromossomopatia segundo o tipo de malformação fetal observada à ultra-sonografia, os dismorfismos faciais apresentaram forte associação com o risco de cromossomopatia quando associados a outros defeitos estruturais fetais. Este estudo encontra-se de acordo com os dados de literatura, que relatam riscos de até $40 \%$ quando ocorrem múltiplas malformações fetais ${ }^{16,17}$.

As anormalias de parede abdominal apresentaram o segundo maior risco de cromossomopatia, principalmente de trissomia do 18, o que também foi observado em outros estudos ${ }^{18,19}$. As cardiopatias, descritas como um dos defeitos congênitos mais comuns ao nascimento ${ }^{20}$, ocuparam o terceiro lugar em risco relativo para anomalia cromossômica. Alguns estudos relatam freqüência de 20 a 65\% de defeitos cromossômicos quando o diagnóstico de cardiopatia é feito intra-úte$\mathrm{ro}^{21,22}$. Neste estudo, a freqüência foi de $30 \%$, com elevado risco para cromossomopatia. As anomalias de pescoço, higroma cístico e edema nucal, ocuparam o quarto lugar para o risco relativo de anomalia cromossômica, com freqüência de cromossomopatia aproximadamente a metade da descrita na literatura ${ }^{10,12}$.

A translucência nucal, considerada método de alta sensibilidade no rastreamento de cromossomopatias, não foi avaliada no estudo, que utilizou apenas fetos com idades gestacionais acima de 14 semanas.

Conforme observado por diversos autores, as anomalias do trato urinário apresentaram uma prevalência estimada de $0,3-0,7 \%$ na vida intrauterina, associando-se a cromossomopatias em torno de $2-33 \%{ }^{7,10,14}$. Em nosso estudo, a freqüência encontrada esteve dentro do esperado, mais próxima ao limite superior, embora tenha ocorrido em um número pequeno de casos.

Os resultados referentes ao crescimento intra-uterino restrito (CIUR) confirmam as observações prévias, com freqüência de anomalia cromossômica em torno de $40 \%$ quando ocorrem malformações associadas ao CIUR ${ }^{12,23}$. Nosso estudo observou que o risco de anomalias cromossô- micas é maior na presença de malformações associadas e de alterações do líquido amniótico.

O risco de cromossomopatia na presença de malformação múltipla foi quatro vezes maior do que quando a anomalia foi isolada, semelhante ao que é descrito por diversos autores ${ }^{2,14,15,24,25}$. Esse resultado sugere duas observações: a primeira é que, uma vez identificada uma anomalia isolada, é extremamente importante procurar com atenção por outras alterações, já que a ocorrência de uma segunda malformação aumenta muito o risco de alteração citogenética. A segunda observação é que a importância do achado da anomalia isolada reside não só no seu próprio valor como achado individual, mas também pela alteração do enfoque dado ao exame, ainda que o diagnóstico se caracterize por uma malformação menor.

Ainda que no Brasil o objetivo do rastreamento pré-natal não possa ser orientado primariamente com o objetivo de interrupção da gestação, atualmente tem-se obtido significativa jurisprudência de autorização judicial para este procedimento em certas situações, especialmente quando indicam impossibilidade de sobrevida pós-natal. Isto reforça a necessidade de buscar o diagnóstico precoce de alterações morfológicas e orientar a busca de alterações citogenéticas. O cariótipo fetal é o padrão-ouro nesta situações. Porém, a descrição dos achados cromossômicos em fetos com morfologia alterada à ultra-sonografia tem permitido que se acrescentem novos e promissores conhecimentos, demonstrando a correspondência entre eles e aumentando a força de associação já sugerida em estudos anteriores.

Este estudo reforça o conceito de que uma avaliação ultra-sonográfica realizada na metade da gravidez, se possivel repetida em pelo menos duas vezes na gestação, é fundamental para que maior número de diagnósticos de situações de risco para cromosomopatias sejam caracterizados.

Embora diversos autores tenham buscado identificar a associação entre os achados morfológicos ultra-sonográficos alterados e a freqüência da ocorrência de cromossomopatias, não encontramos na literatura latino-americana nenhum estudo com a proposta de avaliar o risco relativo específico para cada anomalia analisada por segmento fetal na triagem de gestações de risco de anomalia cromossômica. Reconhecemos que para uma boa capacidade de generalização dos resultados obtidos seria necessário realizar estudos com uma amostra populacional realmente advinda de uma população de baixo risco. Entretanto, há limitações éticas e clínicas para este tipo de estudo, pois o estudo do cariótipo fetal exige procedimentos invasivos e estes não são isentos de risco para a gestação. O estudo pós-natal do 
cariótipo poderia compensar diminuindo o risco de perda fetal, porém haveria provável redução no diagnóstico dos fetos portadores de cromossomopatias, por sua maior probabilidade de óbito intraútero, exceto para serviços onde se realiza coleta sistemática de material dos óbitos fetais ${ }^{2,7,26}$.

Este estudo permitiu observar que a ultrasonografia apresentou bom desempenho na triagem de gestações de risco para cromossomopatia, com sensibilidade de $97,5 \%$ e especificidade de $39,8 \%$. A prevalência de cromossomopatias na população estudada foi de $9,2 \%$, com um VPP do exame de $14 \%$ e VPN de 99,3\%. Os maiores riscos de cromossomopatia foram estimados para as anomalias de face, parede abdominal, cardiovascular, pescoço e membros. Alterações de pulmão, trato urinário, SNC, pescoço e trato gastrointestinal tiveram risco relativo elevado, entre 23 e 32 . Para os diagnósticos de alterações de coluna, genitália e músculo-esqueléticas, foram poucos os casos, não sendo possivel o cálculo do risco relativo. Quando ocorreram anomalias múltiplas, o risco de cromossomopatia foi quatro vezes maior do que quando a anomalia morfológica foi isolada.

\section{ABSTRACT}

Purpose: to estimate the performance of ultrasound to detect gestations at risk for fetal chromosomal abnormalities.

Methods: four hundred and thirty-six patients selected for the study had undergone ultrasound examination and fetal karyotyping, between March 1993 and March 1998. Two hundred and seventy-seven patients had fetal karyotype for fetal malformation detected on ultrasound and 158 for parental anxiety with normal ultrasound examination. Ultrasound sensitivity and specificity were calculated using fetal karyotype as gold standard. The relative risk for each chromosomal abnormality was calculated according to the altered system on ultrasound examination and the risks of the presence of one or more abnormalities on ultrasound, using the Epi-Info 6.0 software package for statistical analysis.

Results: the relative risks for chromosomal abnormalities were 89 for face malformations, 53 for abdominal wall and cardiovascular, 49.6 for neck, 44.6 for extremities, 42.4 for lung, 32.7 for gastrointestinal tract, 27.4 for central nervous system and 23.0 for urinary tract malformations. The relative risk for fetal chromosomal anomalies for genital, thorax, spine and muscle and/or skeletal malformations was not appropriate for calculation because they occurred at very low frequencies. An isolated malformation detected by ultrasound is associated with a 7.8 times higher relative risk for chromosomal anomalies than none, and associated morphologic malformations have a 33.8 times higher relative risk for chromosomal abnormalities.
Conclusion: ultrasound has good performance to detect gestations at risk for chromosomal abnormalities.

KEY WORDS: Ultrasound. Fetal chromosomal abnormalities. Prenatal diagnosis. Karyotype. Fetal malformation.

\section{Referências}

1. Pretorius DH, Nyberg DA. An overview of congenital malformations. In: Nyberg DA, Mahony BS, Pretorius DH, editors. Diagnostic Ultrasound of Fetal Anomalies. $4^{\text {th }}$ ed. Chicago: Year Book Medical Publishers; 1990. p.21-37.

2. Cisneros GLF, Bailão LA, Pina-Neto JM. Estudo genético-clínico das malformações fetais diagnosticadas por ultra-sonografia pré-natal: estimativa da sensibilidade e especificidade. Progr Diagn Prenat 1997; 9:200-8.

3. Castilla EE, Villalobos H, Orioli-Parreiras I, Paz JE. Estudio de malformaciones asociadas. I. Epidemiologia. $1^{\text {a }}$ ed. Maracaibo: Universidad de Zulia/Fondo de Publicaciones de La Unidad de Genética Médica; 1977. p.80-1.

4. Belmonte PL, Sfendrych RR, Sanchez RC, Isfer EV. Ultra-sonografia obstétrica morfológica. RBM Ginecol Obstet 1996; 7:328-36.

5. Penchaszdeh VB. Genética y salud pública. Bol Oficina Sanit Panam 1993; 115:1-11.

6. Cluckle HS, Wald NJ, Lindenbaum RH. Maternal serum alpha-fetoprotein measurement: a screening test for Down syndrome. Lancet 1984; 1:926-9.

7. Carrera JM, Torrents M, Muñoz A, et al. Control ecográfico sistemático para el diagnóstico de malformaciones fetales: 24 años de experiencia. Progr Diagn Prenat 1994; 6: 379-89.

8. Clerici G, Donti E, Zacutti A, Di-Renzo CG. Prenatal diagnosis in Italy. Eur J Hum Genet 1997; 5 Suppl $1: 42-7$

9. Benacerraf BR, Frigoletto FD Jr, Greene MF. Abnormal facial features and extremities in human trisomy syndromes: prenatal US appearance. Radiology 1986; 159:243-6.

10.Bajo JM, Haya FJ. Indicadores ultrasonográficos de técnicas de cariótipo. Progr Diagn Prenat 1994; 6: 51-62.

11.Babcook CJ, Goldstein RB, Filly RA. Prenatally detected fetal myelomeningocele: is karyotype analysis warranted? Radiology 1995; 194:491-4.

12.Carrera JM, Torrents M, Devesa R, Cos T. Screening prenatal ecográfico de las anomalias cromosómicas. Progr Diagn Prenat 1995; 7: 281-90. 
13.Daker M, Bobrow M. Effective care in pregnancy and childbirth. $2^{\text {nd }}$ ed. Oxford: University Press; 1991. p.366-81.

14.Nicolaides KH, Snijders RJ, Gosden CM, Berry C, Campbell S. Ultrasonographically detectable markers of fetal chromosomal abnormalities. Lancet 1992; 340:704-7.

15.Plaza FJ, Olaizola I, De La Fuente P. Eficácia del diagnóstico ecográfico de screening en las malformaciones fetales. Progr Diagn Prenat 1995; 7: 573-8.

16.Saltzman DH, Benaceraff BR, Frigoletto FD Jr. Diagnosis and management of fetal facial clefts. Am J Obstet Gynecol 1986; 155:377-9.

17.Levi S. Relación costo-eficacia del screening prenatal. Progr Diagn Prenat 1996; 8: 384-418.

18.Fink IJ, Roy RA. Omphalocele associated with umbilical cord allantoic cyst-sonographic evaluation in utero. Radiology 1983; 149:473-6.

19.Jauniaux E, Donner C, Thomas C, Francotte J, Rodesch F, Avni FE. Umbilical cord pseudocyst in trisomy 18. Prenat Diagn 1988; 8:557-63.

20.Isfer EV, Sanchez RC, Saito M. Ultra-sonografia e sua importância na medicina fetal. In: Medicina fetal: diagnóstico e conduta. $1^{\text {a }}$ ed. Rio de Janeiro: Revinter, 1996; p.72-82.
21.Copel JA, Cullen M, Green JJ, Mahoney MJ, Hobbins JC, Kleinman CS. The frequency of aneuploidy in prenatally diagnosed congenital heart disease: an indication for fetal karyotyping. Am J Obstet Gynecol 1988; 158:409-13.

22.Nicolaides KH, Shawwa L, Brizot ML, Snijders RJM. Ultrasonographically detectable markers of fetal chromosomal defects. Ultrasound Obstet Gynecol 1993; 3:59-9.

23. Benacerraf BR, Miller WA, Frigoletto FD Jr. Sonographic detection of fetuses with trisomies 13 and 18: accuracy and limitations. Am J Obstet Gynecol 1988; 158:404-9.

24.Benacerraf BR, Neuberg D, Frigoletto FD Jr. Humeral shortening in second trimester fetuses with Down syndrome. Obstet Gynecol 1991; 77:2237.

25.Benacerraf BR, Neuberg D, Bromley B, Frigoletto FD Jr. Sonographic scoring index for prenatal detection of chromosomal abnormalities. J Ultrasound Med 1992; 11:449-58.

26.Weston MJ, Porter HJ, Andrews HS, Berry PJ. Correlation of antenatal ultrasonography and pathological examinations in 153 malformed fetuses. J Clin Ultrasound 1993; 21:387-92.

Recebido em: 17/08/01 Aceito com modificações em: 29/01/02

\section{RBGO}

\section{é uma publicação da FEBRASGO}

\section{que aceita artigos provenientes de} ginecologistas, obstetras e de outras especialidades. Portanto, publique!!! Mande já seu artigo para RBGO 